\title{
Prognosis and recovery in ischaemic and traumatic spinal cord injury: clinical and electrophysiological evaluation
}

\author{
Esther Iseli, Arno Cavigelli, Volker Dietz, Armin Curt
}

\begin{abstract}
Objectives-To compare prognostic factors and functional recovery between paraplegic patients with either ischaemic (28 patients) or traumatic (39 patients) spinal cord injury (SCI).
\end{abstract}

Methods-On admission to the spinal injury centre and 6 months later the patients underwent clinical (following the guidelines set down by the American Spinal Injury Association) and electrophysiological (tibial and pudendal somatosensory evoked potentials) examinations in parallel. The degree of ambulatory capacity was assessed after discharge from the rehabilitation programme or at least 6 months after trauma.

Results-At the acute stage of either ischaemic or traumatic SCI similar motor and sensory deficits and pathological SSEP recordings were present. Both patient groups recovered to similar degrees with respect to motor, sensory, and ambulatory capacity. The clinical examination in both patient groups was the most sensitive prognostic factor of functional recovery irrespective of the aetiology of the SCI. In the ischaemic patients only the tibial SSEP whereas in the traumatic patients both the pudendal and tibial SSEP were of value in predicting recovery.

Conclusions-Although the two patient groups are pathophysiologically different, the severity and extent of neurological deficits and rate of recovery are quite similar. In both ischaemic and traumatic SCI clinical and electrophysiological examinations are of prognostic value for the functional recovery.

(F Neurol Neurosurg Psychiatry 1999;67:567-571)

Swiss Paraplegic

Centre, University

Hospital Balgrist,

Zürich, Switzerland

E Iseli

A Cavigelli

V Dietz

A Curt

Correspondence to:

Dr Armin Curt, University

Hospital Balgrist,

Forchstrasse 340, CH 8008, Zürich, Switzerland.

Telephone 0041 13863904 ; fax 004113863909 ; email

acurt@balgrist.unizh.ch

Received 15 October 1998 and in revised form 18 February 1999

Accepted 23 February 1999 the first and most important approach in the diagnostic assessment of the SCI is the clinical examination of the patient. The American Spinal Injury Association protocol (ASIA) provides a standardised assessment of the extent and severity of neurological deficits based on motor and sensory scores. ${ }^{4}$ Its value in predicting ambulatory recovery has been shown previously in patients with traumatic SCI. ${ }^{5}$ Clinical examination is of limited value in uncooperative patients (due to trauma, drugs, psychiatric disorders etc), electrophysiological recordings such as the somatosensory evoked potentials (SSEPs) can be used supplementary to determine the extent and severity of the spinal cord lesion in such patients. ${ }^{6}$ In patients with SCI the prognosis of the outcome of ambulatory capacity is of crucial importance for clinical and rehabilitative purposes as it may determine if the patients will be wheelchair bound or not. ${ }^{7}$

The aim of this study was to evaluate the prognostic importance of the clinical examination compared with electrophysiological recordings with respect to the extent of functional recovery of patients with ischaemic and traumatic SCI. This was done by comparing neurological deficits and electrophysiological recordings in the acute stages of SCI with those at least 6 months after the injury. Therefore, those indices recorded in the first examination which indicate best the recovery of locomotor function can be determined.

\section{Methods}

PATIENTS WITH ISCHAEMIC SCI

In a retrospective study medical records of 28 patients with acute ischaemic myelopathic SCI (due to non-traumatic and non-compressive factors) were analysed. The 28 patients had been admitted to our centre between March 1985 and 1997 representing about $4.2 \%$ (28 of 667 patients) of all our admissions for primary rehabilitation of SCI. This group consisted of four female and 24 male patients with levels of lesion C6 - L2. The causes of ischaemic myelopathy were due to aortic aneurysm repair $(\mathrm{n}=12$ patients), arteriovenous malformation $(\mathrm{n}=2)$, ischaemia during scoliosis/ kyphosis correction $(n=3)$, and spinal hypotension due to intoxocation $(n=2)$. In the remaining nine patients the spinal cord lesion was attributed to ischaemia on the basis of anamnesis (for example, sudden onset). Other possible causes were ruled out by various examinations including radiography, CT, MRI, or CSF examination. 
Clinical data and mean ASIA scores (95\% confidence interval) of the patients with ischaemic and traumatic spinal cord lesion

\begin{tabular}{|c|c|c|c|c|c|c|c|c|}
\hline \multirow[b]{3}{*}{ Age† } & \multicolumn{4}{|c|}{ Ischaemic lesions } & \multicolumn{4}{|c|}{ Traumatic lesions } \\
\hline & \multicolumn{2}{|c|}{ Initial $(n=28)$} & \multicolumn{2}{|c|}{ After 6 months $(n=18)$} & \multicolumn{2}{|c|}{ Initial $(n=39)$} & \multicolumn{2}{|c|}{ After 6 months $(n=39)$} \\
\hline & 56.07 & $(48.67-63.47)$ & & & 40.01 & $(34.57-45.43$ & & \\
\hline ASIA motor score & 57.22 & $(51.35-63.1)$ & $62.21^{\star}$ & $(53.92-70.5)$ & 59.92 & $(55.56-64.28)$ & $67.58^{\star}$ & $(61.97-73.19)$ \\
\hline ASIA light touch score & 75.26 & $(65.3-85.22)$ & $85.01^{\star}$ & $(72.27-97.73)$ & 86.21 & $(80.88-91.53)$ & $88.76^{\star}$ & $(83.42-94.11)$ \\
\hline ASIA pin prick scoret & 65.85 & $(57.99-73.72)$ & 69.37 & $(59.54-79.21)$ & 82.46 & $(76.67-88.25)$ & $86.89^{\star}$ & $(80.86-92.93)$ \\
\hline
\end{tabular}

^Significant increment 6 months after admission.

†Significant difference between the two patient groups.

PATIENTS WITH TRAUMATIC SCI

The traumatic SCI patients, studied prospectively, comprised 39 patients (nine female and 30 male) with levels of lesion between T1 - L5, who were admitted to hospital for primary rehabilitation between 1992 and 1994.

In both patient groups accompanying nontraumatic diseases of the peripheral nervous system (polyneuropathy, nerve entrapments) and cerebral lesions were excluded by clinical and electrophysiological examinations. All patients were informed about the aim of the study and gave their consent to participation. In addition, any patient was allowed to drop out of the study at any time if they wished.

\section{CLINICAL ASSESSMENT OF AMBULATORY}

CAPACITY

The ambulatory capacity of the patients was attributed to one of four categories: (1) no ambulatory capacity-the patient could neither walk nor stand; (2) therapeutic ambulatory capacity - standing and walking only by support of two physiotherapists, or with two braces in parallel bars, or with two sticks and the help of an accompanying person (the patient can perform these procedures only as a therapeutic approach); (3) functional ambulatory capacity-walking daily over short distances without external personal support, while the use of one or two sticks or braces is allowed (the ambulatory capacity is regularly used in the activities of daily living and is usable for employment); (4) full ambulatory capacity-little or no disturbance in walking. This assessment corresponds to those used in earlier studies. ${ }^{5}$ The outcome of ambulatory capacity was assessed in the chronic stage of spinal cord injury at least 6 months after injury. The categorisation was focused on both the walking level achieved and the walking activities regularly practised at home by the patient.

\section{CLINICAL EXAMINATION}

Neurological examination of all patients was performed according to the 1992 ASIA standards. ${ }^{4}$ Two examinations were performed, the first after admission to our rehabilitation centre and the second 6 months later. Within the ASIA protocol the following indices were evaluated: (1) motor score, (2) pinprick score, (3) light touch score, and (4) the differentiation between complete or incomplete lesion. The neurological examination was performed by full time specially trained physicians who were all familiar with the ASIA protocol.

\section{ELECTROPHYSIOLOGICAL RECORDINGS}

The tibial SSEPs were elicited by electrical stimulation (square wave of $0.2 \mathrm{~ms}$ duration applied at $3 \mathrm{~Hz}$ ) of the tibial nerves of both legs, at the medial ankle with the cathode placed 2 to $3 \mathrm{~cm}$ proximal to the anode. The stimulus intensity (up to a maximum of 40 $\mathrm{mA}$ ) was adjusted to produce a clear muscle contraction or sensation. The pudendal SSEPs were elicited by the same stimulation indices applied via ring electrodes on the penis for men and skin electrodes at the level of the clitoris in women as described elsewhere. ${ }^{9}{ }^{10}$ Patients were lying prone at ambient room temperature. The recording electrodes (conventional $0.5 \mathrm{~cm}$ gold cup electrodes) were attached to the skin over the popliteal fossa for tibial SSEPs (to control the effectiveness of the electrical stimulation and transmission of the potentials along the peripheral nerve segments). Scalp electrodes were positioned at $\mathrm{Cz}$ '-Fz (international $10 / 20$ electrode system). The electrode impedance was maintained at $<5 \mathrm{k} \Omega$. The amplifier was set at $5 \mu \mathrm{V} /$ division and the time of analysis at $60 \mathrm{~ms}$. Two sets of 500 responses were averaged and superimposed to ensure consistency. Electrophysiological recordings of a reference group of 10 healthy subjects (five men and five women; mean age 29.6 years, range 24 to 38 years) described previously were used for comparison. ${ }^{5}$

\section{STATISTICS}

Statistical evaluations were performed using an SAS software package. Student's $t$ test was used to compare the values of the two patient groups. The increments of the ASIA scores were analysed with the paired Student's $t$ test. The $\chi^{2}$ test was used to determine the differences between complete and incomplete lesions according to the outcome of ambulatory capacity. Spearman's rank correlation coefficients were calculated to determine the prognostic value of age, ASIA scores, and electrophysiological recordings for ambulatory capacity. To determine the best combination of all mentioned variables for the prediction of ambulatory capacity a stepwise multiple regression analysis was used (significance level for entry into the model was set to 0.15). Analysis of variance (ANOVA) and Scheffe's test for multiple pairwise comparisons were used to compare the SSEP recordings of the two patient groups and the control group. For all statistical tests and correlations $\mathrm{p}<0.05$ was considered as significant and only natural values of the electrophysiological recordings were calculated. 

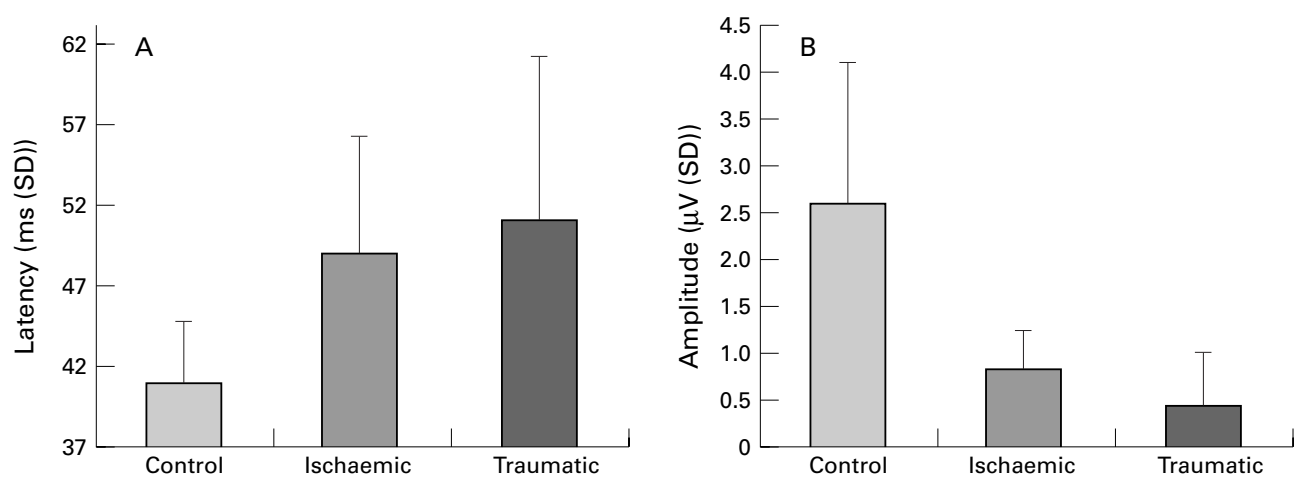

Figure 1 Values (mean (SD)) of tibial SSEP latency $(A)$ and amplitude (B) from healthy subjects (controls) and both patient groups.

\section{Results}

PATIENTS

The mean age of the patients with ischaemic lesions (56.1 (SD 19.1) years) was significantly ( $t$ test, $\mathrm{p}<0.001$ ) higher than that of the patients with traumatic lesions (40.0 (SD 16.8) years, table). Clinical differentiation into complete or incomplete SCI using the ASIA protocol showed that the complete to incomplete ratio was similar $\left(\chi^{2}\right.$ test, $\left.p>0.05\right)$ in both patient groups (ischaemic lesions 1:1.33, traumatic lesions 1:1.29).

NEUROLOGICAL DEFICIT AND RECOVERY

The initial clinical examination after admission to the rehabiliation centre was performed in the ischaemic patient group between 0 to 30 days (mean 2.1 days) and in the traumatic injury patient group between 1 to 35 days (mean 10.0 days) after incidence of paraplegia. Both patient groups showed similar values for the motor and light touch scores (table). The pinprick scores were significantly lower in the ischaemic than in the traumatic injury patients ( $t$ test, $\mathrm{p}<0.0007)$. Follow up recordings taken 6 months later showed that all the ASIA indices had increased significantly in the traumatic injury patients ( $t$ test, motor score: $\mathrm{p}<0.0001$; light touch score: $\mathrm{p}<0.007$; pinprick score: $\mathrm{p}<0.005)$. In the ischaemic patient group only the motor and light touch scores increased $(t$ test, motor score $\mathrm{p}<0.007$; light touch score $\mathrm{p}<0.03$; pinprick score $\mathrm{p}>0.20$ ). The extent of neurological recovery, calculated by the increment of ASIA scores, was similar in both patient groups ( $t$ test, $\mathrm{p}>0.3$ ).

SSEP RECORDINGS

The SSEP recordings were performed in parallel to the clinical examinations. In both groups, the mean amplitude of the tibial SSEP (trauma $\mathrm{n}=39$, amplitude $0.42(\mathrm{SD} 0.56) \mu \mathrm{V}$; ischaemia $\mathrm{n}=24$, amplitude $0.77(\mathrm{SD} 0.46) \mu \mathrm{V})$ was significantly reduced compared with the control group (amplitude 2.6 (SD 1.54) $\mu \mathrm{V}$; Scheffe's test, $\mathrm{p}<0.05$, fig 1 ). There was no significant difference of impairment between the two patient groups. ANOVA testing of the tibial SSEP latency values of the control group (latency 40.99 (SD 3.8) $\mathrm{ms}$ ) and the two patient groups (traumatic: 50.83 (SD 10.27) $\mathrm{ms}$; ischaemic: 48.8 (SD 7.3) ms) indicated a significant difference between the healthy sub-

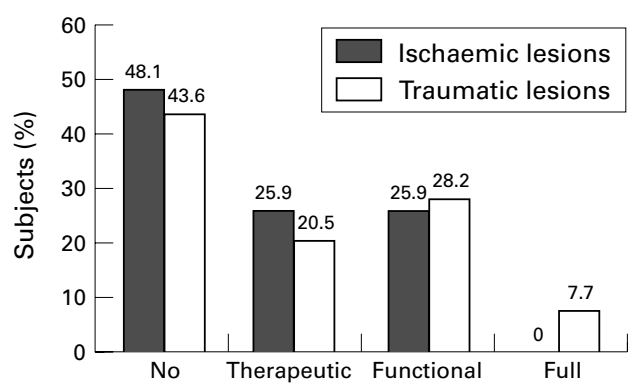

Figure 2 Outcome of ambulatory capacity at discharge from the rehabilitation programme in both patient groups.

jects and the patients $(p<0.05)$. A further analysis with Scheffe's test showed a tendency for prolonged tibial latencies of the traumatic injury compared with the ischaemic patients $(\mathrm{p}<0.06)$. The recordings of the pudendal SSEPs of the two patient groups showed similar amplitudes (traumatic injury $n=33$, amplitude 0.33 (SD 0.43) $\mu \mathrm{V}$; ischaemia $\mathrm{n}=18$, amplitude 0.22 (SD 0.3) $\mu \mathrm{V} ; \mathrm{p}>0.3$ ), and latencies (traumatic injury: 53.68 (SD 15.10) ms; ischaemic: 50.77 (SD 13.25) ms; $\mathrm{p}>0.1$ ).

RECOVERY OF AMBULATORY CAPACITY

The degree of recovery of ambulatory capacity determined at discharge from the rehabilitation programme or after at least 6 months, was similar in both the ischaemic and traumatic injury groups $\left(\chi^{2}\right.$ test, $\left.p>0.05\right)$. Both patient groups still had severe impairment of lower limb function. Only about $25 \%$ of the ischaemic patients achieved a functional ambulatory capacity whereas about $35 \%$ of traumatic injury patients recovered at least functional ambulatory capacity (fig 2).

\section{PROGNOSIS OF AMBULATORY CAPACITY}

The age of the patients when SCI occurred was of no prognostic value for the recovery of ambulatory capacity (ischaemic $r=-0.14$, $\mathrm{p}>0.05$; traumatic injury $r=-0.19, \mathrm{p}>0.05)$.

The clinical differentiation into complete or incomplete SCI lesion was indicative of recovery of ambulatory capacity in traumatic injury patients only ( $\chi^{2}$ test, $\left.\mathrm{p}<0.001\right)$. Only $12.5 \%$ of the initially complete traumatic injury patients regained some ambulatory capacity at the end of the rehabilitation, whereas about $35 \%$ of the initially complete ischaemic patients recovered some ambulatory function. 


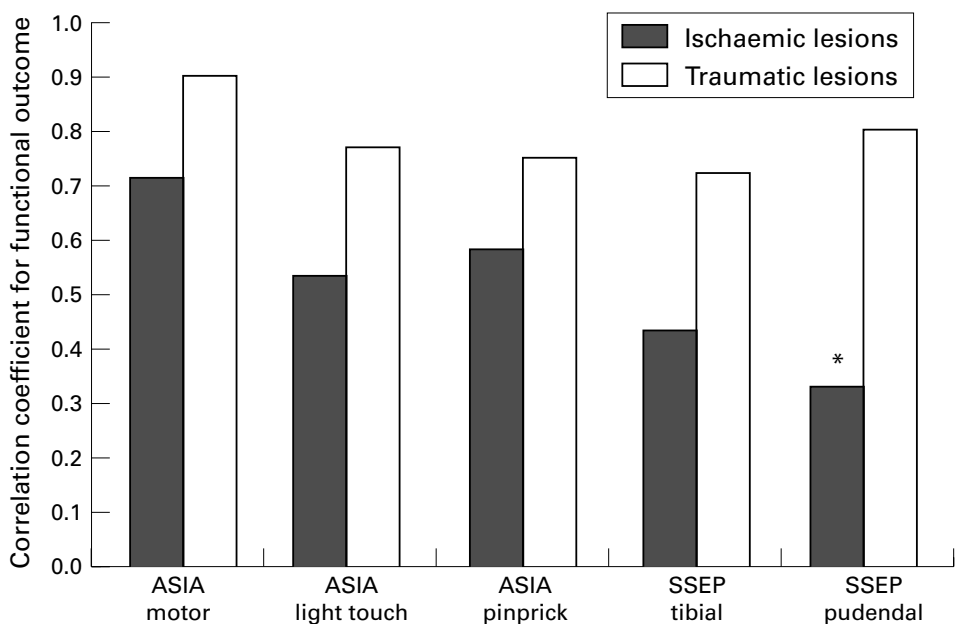

Figure 3 Correlation (Spearman's rank correlation) between clinical and neurophysiological indices and outcome of ambulatory capacity (* not significant).

The initial ASIA motor and sensory scores were strongly indicative of recovery in both patient groups (fig 3). The best prognostic factor was the ASIA motor score (ischaemic patients $r=0.71, \mathrm{p}<0.0001$; traumatic injury patients $r=0.90, \mathrm{p}<0.0001)$.

Similarly to the clinical examination the tibial SSEP recordings were also strongly indicative for the degree of ambulatory recovery (traumatic injury patients $r=0.72$, $(\mathrm{p}<0.00001)$; ischaemic patients $r=0.43$, $(\mathrm{p}<0.04))$. The pudendal SSEP recordings were significantly related to the ambulatory capacity but only in the traumatic injury patients $(r=0.80, \mathrm{p}<0.0001)$. For the ischaemic patients the correlation coefficient was 0.33 $(p>0.17)$. In general, the SSEP recordings were of less prognostic value in the ischaemic compared with the traumatic injury patients. Of all the clinical and electrophysiological indices the best prediction of outcome of ambulatory capacity was achieved by the combination of the motor score and tibial SSEP recordings in both patient groups (multiple regression analysis, $\mathrm{R}^{2}=0.72$ ).

\section{Discussion}

In several studies on traumatic SCI patients the extent of neurological deficits, follow up examinations of neurological recovery, functional outcome, and prognostic factors have been evaluated by an established clinical scoring system (ASIA protocol). ${ }^{811}$ However, this analysis has not been extended to patients with ischaemic SCI. Therefore, this study compared patients with ischaemic or traumatic SCI using established, standardised clinical and electrophysiological examinations.

The main findings were: (1) both patient groups (ischaemic and traumatic injury) showed a similar extent of neurological deficit when assessed using both clinical examination (ASIA protocol) and pathological SSEP recordings. (2) There was no significant difference between the groups in the degree of neurological recovery of motor and sensory function after the injury. (3) The patients of both groups recovered ambulatory capacity to similar degrees. (4) The clinical examination and the SSEP recordings in both acute traumatic and ischaemic SCI were of significant value in predicting the extent of functional recovery.

In patients with acute SCI the clinical examination is the first and most important approach in the diagnostic assessment of a spinal cord lesion. ${ }^{45}$ Several studies on patients with traumatic SCI have shown that the clinical evaluation of muscle strength and pinprick sensation can predict the outcome of ambulatory capacity with an accuracy of about $80 \% .{ }^{13}$

The ASIA protocol describes a standardised clinical examination of motor and sensory functions in cases of traumatic SCI. This ensures not only accurate communication between clinicians but also direct comparison between different research studies. However, the ASIA protocol has not yet been evaluated for patients with ischaemic SCI. Furthermore, no comparative study between acute traumatic and ischaemic spinal cord lesions has been described which uses a standardised clinical examination protocol.

Both patient groups showed similar degrees of neurological deficit on admission and also 6 months later. The ischaemic patients showed the same degree of neurological recovery as patients with traumatic SCI despite the fact that they were generally older and the aetiology of the SCI was totally different. According to earlier reports the ischaemic lesion often arose due to complications after aortic surgery, other conditions producing spinal hypotension, or was associated with general disorders of blood circulation such as atheriosclerosis. ${ }^{14-17}$ In agreement with other studies, about $30 \%$ of the ischaemic patients used in this study were suspected of having ischaemic lesions simply by exclusion of other disorders. ${ }^{18} 19$

Electrophysiological recordings provide supplementary information to the clinical examination and are of significant value in uncooperative patients. $^{20-22}$ Furthermore, electrophysiological recordings can be used to determine accompanying lesions of the peripheral nervous system, which are difficult to assess in acute SCI by clinical examination. ${ }^{22}$ The present study showed that pudendal and tibial SSEP recordings are severely affected in both ischaemic and traumatic SCI patients. The SSEP amplitudes were significantly reduced in both groups of SCI patients, whereas the SSEP latencies were more prolonged in traumatic compared with ischaemic patients. This may be due to the different underlying pathophysiological mechanism as an ischaemic injury affects primarily the central axonal and neuronal structures of the spinal cord-that is, it induces less demyelination than a traumatic lesion. This would be in line with the findings that pinprick sensations and pudendal SSEPs were more affected in ischaemic than in traumatic injury patients. The latter indices represent a predominant lesion of the grey matter.

An early prognosis of the functional recovery, such as the recovery of ambulatory capacity, is essential in acute SCI patients to efficiently plan physical and functional training as well as home adaptations. ${ }^{11}$ Therefore, ambulatory capacity assessed 6 months after the initial injury was differentiated into four 
categories with respect to the activities of daily living. ${ }^{20}$ Several studies have documented a positive correlation of ASIA scores with the outcome of ambulatory capacity in patients with traumatic SCI. ${ }^{523}$ Our results proved that this correlation is also true for patients with ischaemic SCI. Although SSEP recordings reflect sensory deficits related to dorsal column function they are of significant prognostic value with respect to ambulatory capacity. It has been shown that tibial and pudendal SSEPs are very strongly associated with the recovery of ambulatory capacity in patients with traumatic SCI. ${ }^{511}$ In the ischaemic patient group significant correlations with the outcome of ambulatory capacity were found only for the tibial SSEPs.

We conclude that although the two patient groups are pathophysiologically different, the severity and extent of neurological deficits and rate of recovery are quite similar. In both ischaemic and traumatic SCI clinical (most sensitive are the ASIA motor scores) and electrophysiological (tibial SSEP) examinations are of prognostic value to predict the functional recovery and thus allow early planning of rehabilitation aims and procedures.

We thank Dr I Gibson for editorial service, E Harsch for technical assistance, and Th Erni (biostatistician) for statistica support. This work was supported by the Swiss National Science Foundation (Grant No 3200-052562.97) and the International Research Institute for Paraplegia (P38/97).

1 Cheshire WP, Santos CC, Massey EW, et al. Spinal cord inf.

2 Satran R. Spinal cord infarction. Stroke 1987;22:13-17.

3 Pelser H, Van Gijn J. Spinal infarction: a follow-up study. Stroke 1993;24:896-8.

4 Ditunno JF, Young W, Donovan WH, et al. The international standards booklet for neurological and functional classification of spinal cord injury. Paraplegia 1994;32:7080
5 Curt A, Dietz V. Ambulatory capacity in spinal cord injury: significance of somatosensory evoked potentials and ASIA protocol in predicting outcome. Arch Phys Med Rehabil 1997;78:39-43

6 Curt A, Dietz V. Traumatic cervical spinal cord injury: relation between somatosensory evoked potentials, neurologi77:48-53.

7 Bednarczyk JH, Sanderson DJ. Comparison of functional and medical assessment in the classification of persons with and medical assessment in the classification of per

8 Donovan WH, Wilkerson MA, Rossi D, et al. A test of the ASIA guidelines for classification of spinal cord injuries. $\mathcal{F}$ Rehabil 1990;4:39-53.

9 Haldeman S, Bradley WE, Bhatia NN, et al. Pudendal evoked responses. Arch Neurol 1982;39:280-3.

10 Curt A, Rodic B, Schurch B, et al. Recovery of bladder function in patients with acute spinal cord injury: function in patients with acute spinal cord injury: potentials. Spinal Cord 1997;35:368-73.

11 Jacobs SR, Yeaney NK, Herbison GJ, et al. Future ambulation prognosis as predicted by somatosensory evoked potentials in motor complete and incomplete quadriplegia. Arch Phys Med Rehabil 1995;76:635-41.

12 Kaplan PE, Rosen JS. Somatosensory evoked potentials in spinal cord injured patients. Paraplegia 1981;19:118-22.

13 Waters RL, Adkins R, Yakura J, et al. Prediction of ambulators performance based on motor scores derived from tors performance based on motor scores derived from standards of the American Spinal Inju
Phys Med Rehabil 1994;75:756-60.

14 Gaeta TJ, LaPolla GA, Balentine JR. Anterior spinal artery infarction. Ann Emerg Med 1995;26:90-2.

15 Foo D, Rossier AB. Anterior spinal artery syndrome and its natural history. Paraplegia 1983;21:1-10.

$16 \mathrm{Kim} \mathrm{SW,} \mathrm{Kim} \mathrm{RC,} \mathrm{Choi} \mathrm{BH,} \mathrm{et} \mathrm{al.} \mathrm{Non-traumatic} \mathrm{ischaemic}$ myelopathy: a review of 25 cases. Paraplegia 1988;26:26272.

17 Silver JR, Buxton PH. Spinal stroke. Brain 1974;97:539-50.

18 Elksnis SM, Hogg JP, Cunningham ME. MR imaging of spontaneous spinal cord infarction. F Comput Assist Tomogr 1991;15:228-32.

19 Hughes JT, Brownell B. Spinal cord ischemia due to arterioclerosis. Arch Neurol 1966;15:189-202.

20 Curt A, Keck ME, Dietz V. Functional outcome following spinal cord injury: significance of motor-evoked potentials and ASIA scores. Arch Phys Med Rehabil 1998;79:81-6.

21 Grundy BL, Friedman W. Electrophysiological evaluation of the patient with acute spinal cord injury. Crit Care Clin 1987;3:519-48

22 Houlden DA, Schwart ML, Klettke KA. Neurophysiologic diagnosis in uncooperative trauma patients: confounding factors. F Trauma 1992;33:244-51

23 Rowed DW, McLean JAG, Tator CH. Somatosensory evoked potentials in acute spinal cord injury: prognostic value. Surg Neurol 1978;9:203-10. 